\title{
WASP-135b: a highly irradiated, inflated hot Jupiter orbiting a G5V star
}

\author{
J. J. Spake ${ }^{1,2}$, D. J. A. Brown², A. P. Doyle 2 , G. Hébrard ${ }^{3,4}$, J. McCormac ${ }^{2}$, D. J. \\ Armstrong $^{2,5}$, D. Pollacco ${ }^{2}$, Y. Gómez Maqueo Chew ${ }^{6}$, D. R. Anderson 7 , S. C. C. Barros ${ }^{8}$, \\ F. Bouchy ${ }^{8}$, P. Boumis ${ }^{9}$, G. Bruno ${ }^{8}$, A. Collier Cameron ${ }^{10}$, B. Courcol $^{8}$, G. R. Davies ${ }^{11,12}$, \\ F. Faedi ${ }^{2}$, C. Hellier ${ }^{7}$, J. Kirk ${ }^{2}$, K. W. F. Lam², A. Liakos ${ }^{9}$, T. Louden², P. F. L. Maxted ${ }^{7}$, \\ H. P. Osborn ${ }^{2}$, E. Palle ${ }^{13,14}$, J. Prieto Arranz ${ }^{13,14}$, S. Udry ${ }^{15}$, S. R. Walker ${ }^{2}$, R. G. West ${ }^{2}$, P. \\ J. Wheatley ${ }^{2}$ \\ jspake@astro.ex.ac.uk
}

\begin{abstract}
We report the discovery of a new transiting planet from the WASP survey. WASP-135b is a hot Jupiter with a radius of $1.30 \pm 0.09 R_{\mathrm{Jup}}$, a mass of $1.90 \pm 0.08 M_{\mathrm{Jup}}$ and an orbital period of 1.401 days. Its host is a Sun-like star, with a G5 spectral type and a mass and radius of $0.98 \pm 0.06 M_{\odot}$ and $0.96 \pm 0.05 R_{\odot}$ respectively. The proximity of the planet to its host means that WASP-135b receives high levels of insolation, which may be the cause of its inflated radius. Additionally, we find weak evidence of a transfer of angular momentum from the planet to its star.
\end{abstract}

Subject headings: extrasolar planets - stars: individual: WASP-135 - techniques: radial velocity, photometry

\footnotetext{
${ }^{1}$ Astrophysics Group, School of Physics, University of Exeter, Stocker Road, Exeter, EX4 4QL, UK

${ }^{2}$ Department of Physics, University of Warwick, Coventry CV4 7AL, UK

${ }^{3}$ Institut d'Astrophysique de Paris, UMR7095 CNRS, Université Pierre \& Marie Curie,98bis boulevard Arago, 75014 Paris, France

${ }^{4}$ Observatoire de Haute-Provence, CNRS, Université d'Aix-Marseille, 04870 Saint-Michel-l'Observatoire, France

${ }^{5}$ ARC, School of Mathematics \& Physics, Queen?s University Belfast, University Road, Belfast BT7 1NN, UK

${ }^{6}$ Instituto de Astronomía, UNAM, 04510, México, D.F., México

${ }^{7}$ Astrophysics Group, Keele University, Staffordshire ST5 5BG, UK

${ }^{8}$ Aix Marseille Université, CNRS, LAM (Laboratoire d'Astrophysique de Marseille) UMR 7326, 13388, Marseille, France

${ }^{9}$ IAASARS, National Observatory of Athens, GR-15236 Penteli, Greece

${ }^{10}$ SUPA, School of Physics \& Astronomy, University of St. Andrews, North Haugh, St. Andrews, Fife KY16 9SS, UK

${ }^{11}$ School of Physics and Astronomy, University of Birm-
}

\section{Introduction}

The discovery of the first hot Jupiter (Mayor \& Queloz 1995) was surprising since no Solar System analogue existed, and gas giants are not expected to form so close to their parent star. They are an intriguing class of exoplanets, and those that transit are important since they remain the only planets for which accurate measurements of both mass and radius can be made. Additionally, they are excellent targets for studying the atmospheric compositions of exoplanets, through transit spec-

ingham, Birmingham, B15 2TT, UK

${ }^{12}$ Stellar Astrophysics Centre (SAC), Department of Physics and Astronomy, Aarhus University, Ny Munkegade 120, DK-8000 Aarhus C, Denmark

${ }^{13}$ Instituto de Astrofisica de Canarias, Via Lactea s/n 38200, La Laguna, Spain

${ }^{14}$ Departamento de Astrofsica, Universidad de La Laguna (ULL), La Laguna, 38205, Tenerife, Spain

${ }^{15}$ Observatoire de Genéve, Université de Genéve, Chemin des mailletes 51, 1290 Sauverny, Switzerland 
troscopy.

There is still much that is not entirely understood about hot Jupiters, such as their formation and dynamical evolution mechanisms, and the fact that many appear to have inflated radii (see Fortney \& Nettelmann (2010), Baraffe et al. (2010) and Spiegel \& Burrows (2013)). It has been suggested that the unexpectedly large radii of some hot Jupiters can be explained by the injection of some of the received stellar energy into the planetary interior (Showman \& Guillot 2002). Indeed there is growing evidence of a positive correlation between levels of incident flux and giant planet radius, for example in Enoch et al. (2011), Demory \& Seager (2011), and Weiss et al. (2013). However, the exact mechanisms which transport the energy into the interior of planets remain disputed. Proposed theories include Ohmic heating (Batygin \& Stevenson 2010) and kinetic heating (Guillot \& Showman 2002), among others.

Some, such as Lanza (2010), suggest that for hot Jupiter host stars, a gyrochronological age significantly younger than age estimates found by other means is evidence of a transfer of angular momentum from the massive, close-in planet to the star, causing tidal 'spin-up'. This process would hinder the normal stellar spin-down mechanisms, leaving the star rotating faster, and appearing younger than expected from gyrochronological models. Some hints of this effect have been seen, for example Pont (2009) finds empirical evidence of faster rotation in stars with closer and more massive planets, and Brown (2014) and Maxted et al. (2015) both find a tendency for planet hosting stars to have younger gyrochronological ages than their isochronal age estimates.

A larger sample of exoplanets is needed in order to investigate the possible spin-up effect of hot Jupiters on their host stars, and to better understand the relationship between incident stellar flux, planet mass and planet radius. These are some of the reasons that the discovery of transiting hot Jupiters continues to be important. We present here the discovery of one such planet, WASP-135b.

\section{Observations}

\subsection{SuperWASP-N Photometry}

WASP-135 (1SWASPJ174908.40+295244.9) is a G5V star with $V=13.28$ and $B-V=$ 0.97 (Zacharias et al. 2013). It was observed by SuperWASP-N at the Observatorio del Roque de los Muchachos on La Palma, Spain from 2004 May 05 to 2010 August 24, and 34699 observations were made in total. The instrument consists of 8 cameras, with Canon 200-mm f/1.8 lenses and $2048 \times 2048 \mathrm{e} 2 \mathrm{~V}$ CCD detectors, each with a field-of-view of $7.8^{\circ} \times 7.8^{\circ}$ and a plate scale of 13 ".7 pixel $^{-1}$, and broadband $\mathrm{V}+\mathrm{R}$ filters. The WASP survey and data reduction procedures are discussed in detail in Pollacco et al. (2006).

An exoplanet candidate with a period of 1.401 days was identified using transit detection methods described in Collier Cameron et al. (2006) and Pollacco et al. (2008). The top panel of Figure 2.3 shows the WASP photometry, folded on the best-fitting orbital period. Additional transit signals were searched for in the WASP data using the method described in Smith et al. (2009) for planet candidates with periods up to 40 days but none were found. Although the WASP data spans over 6.5 years, systematic noise limits the search for periodicity on very long timescales. The WASP data was also searched for photometric variability as could be caused by magnetic activity and stellar rotation, as described in Maxted et al. (2011). No significant signals were found above the 3 -mmag level.

Before analysis, the timings for all data including WASP photometry were converted to $\mathrm{BJD}_{\mathrm{TDB}}$ using the method of Eastman et al. (2010).

\subsection{SOPHIE Radial Velocities}

Spectroscopic follow-up of WASP 135 was conducted with the SOPHIE spectrograph mounted on the $1.93 \mathrm{~m}$ telescope of the Observatoire de Haute-Provence, France. More details about the instrument can be found in Bouchy et al. (2009).

Measurements were made of the radial velocity of the star at many points throughout the proposed orbital phase. In the case of a planetary system, the signal should describe a Keplerian motion of the planet host around the system's centre of mass, yielding the planetary mass and orbital 

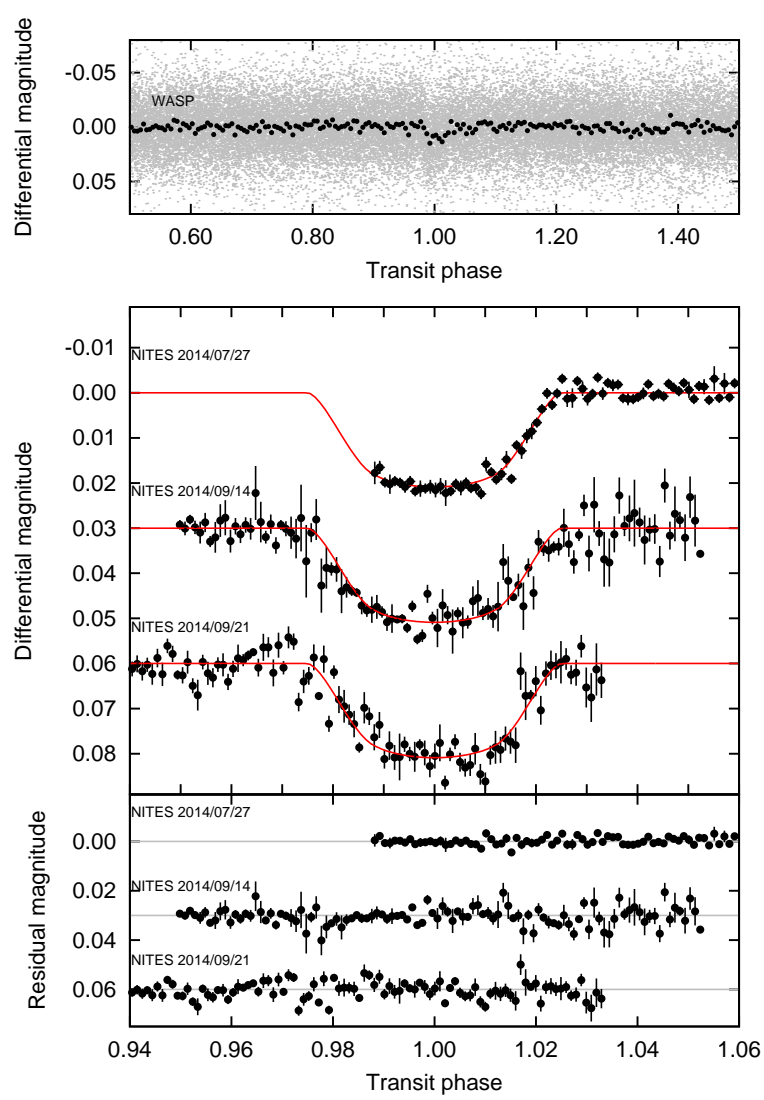

Fig. 1. - Top panel: WASP data (through broadband $\mathrm{V}+\mathrm{R}$ filters) phase-folded on period 1.40138 days, with over-plotted bins of width $\sim 7$ minutes. Lower panels: binned follow-up photometry from NITES (no filter used) with residuals, bin widths $\sim 3$ minutes, over plotted with the best fitting transiting planet model using the formalism of Mandel \& Agol (2002).

eccentricity, among other parameters. The radial velocity measurements also allow us to discard astrophysical false positives such as blended stellar binaries.

SOPHIE was used in High-Efficiency mode (HE) with a resolving power $R=40000$ to increase the throughput for this faint target. The typical exposure times were 30 minutes, but they were slightly adjusted as a function of the weather conditions to keep the signal-to-noise ratio at $\sim 30$. The spectra were extracted using the SOPHIE pipeline, and the radial velocities were measured from the weighted cross-correlation with a numerical mask (Baranne et al. 1996; Pepe et al. 2002). We adjusted the number of spectral orders used in the cross-correlation to reduce the dispersion of the measurements. Indeed, some spectral domains are noisy (especially in the blue part of the spectra), so using them degrades the accuracy of the radial velocity measurement.

The error bars on the radial velocities were computed from the cross-correlation function (CCF) using the method presented by Boisse et al. (2010). We estimated the moonlight contamination using the second SOPHIE fiber aperture, which is targeted on the sky while the first aperture points toward the target. We found RV shifts due to Moonlight contamination below $10 \mathrm{~ms}^{-1}$ in all cases, and concluded that there is no significant Moon pollution in our data. Radial velocities measured using different stellar masks (F0, G2, K0, or K5) produce variations with similar amplitudes, so there is no evidence that their variations could be explained by blend scenarios caused by stars of different spectral types.

In total 21 observations were made between 2014 May 30 and 2014 October 02. These are reported in Table 1 and displayed in Figure 2 together with the Keplerian fit and the residuals. They show variations in phase with the SuperWASP transit ephemeris and with a semiamplitude of a few hundred $\mathrm{ms}^{-1}$, implying a companion of $\sim 2 M_{\text {Jup }}$.

The bisector spans as defined by Toner \& Gray (1988) were measured, and applied to the crosscorrelation function as in Queloz et al. (2001). They show neither variations nor trends as a function of radial velocity or orbital phase (Fig. 3). Additionally, the RMS of the bisector spans is $0.05 \mathrm{kms}^{-1}$, which is of the order of the radial velocity errors. This reinforces the conclusion that the radial-velocity variations are not caused by spectral-line profile changes attributable to blends or stellar activity.

Two radial velocity measurements were not included in the final analysis and hence do not appear in the figure, although they are included Table 1 for completeness. One (BJD = 2456923.4) was particularly inaccurate due to poor weather conditions. The other (BJD $=2456813.4$ ) is an outlier, and is off by about $100 \mathrm{~m} / \mathrm{s}$ from the Keplerian model. We looked for possible causes of this 
shift, including cosmic rays on the detector, abnormally high drift seen in the wavelength calibration, concern with the CCD reading mode, standard stars observed at the same epoch, sporadic stellar activity events linked to CaII chromospheric emission; we found nothing abnormal. However, a more careful inspection of the CCF shows a small deformation of its profile; it is also seen to have a bisector value slightly lower than values at other epochs. Even if the cause of the CCF deformation is not well understood, it explains the abnormal radial velocity at this epoch. This reinforces its interpretation as an instrumental outlier instead of a signature of an additional companion in the system, in agreement with the fact that other RV measurements secured at similar epochs or similar orbital phase do not show such shifts from the 1-planet Keplerian model.

We thus conclude that our target harbours a transiting giant planet, which we designate WASP-135b hereafter.

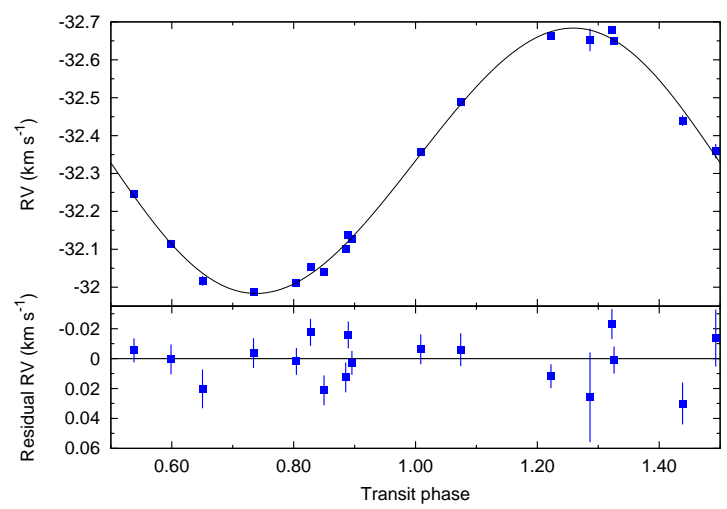

Fig. 2.- All SOPHIE radial velocity data, with the best fitting Keplerian model over plotted. The variations in phase with the transit photometry suggest a sub-stellar companion of $\sim 2 M_{\text {Jup }}$.

\subsection{NITES Photometry}

Three nights of follow-up observations were carried out with the Near Infra-red Transiting ExoplanetS (NITES) telescope on 2014 July 27, 2014 September 21 and 2014 September 28. NITES is a $0.4 \mathrm{~m}, \mathrm{f} / 10$ Meade telescope located at the Observatorio del Roque de los Muchachos on La Palma, equipped with a camera containing a $1024 \times 1024$
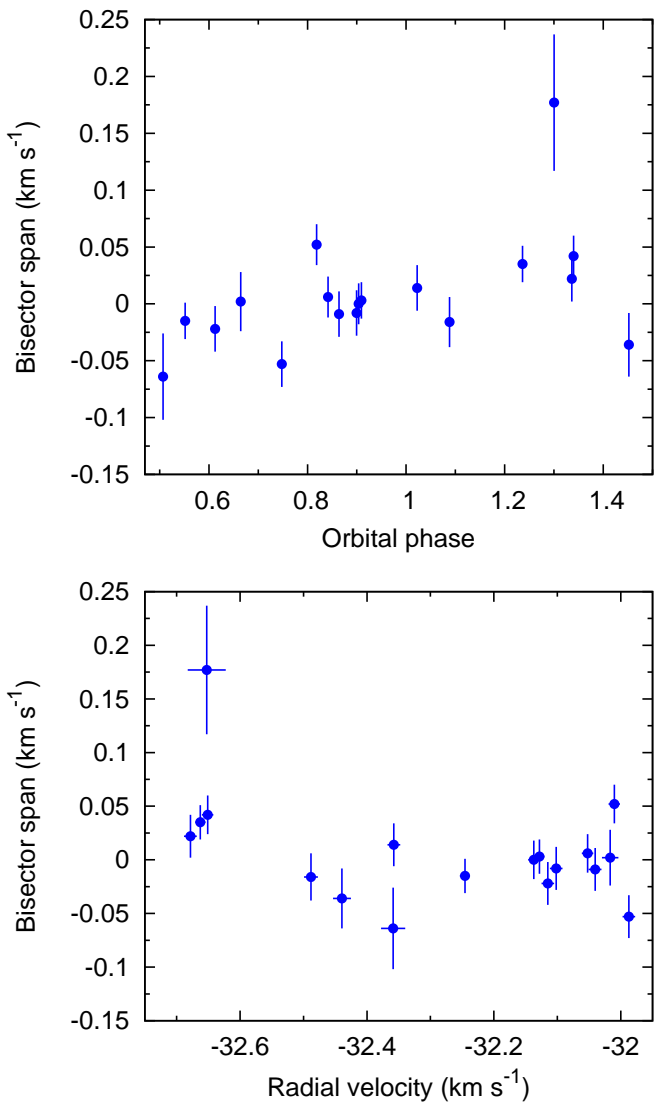

Fig. 3.- CCF bisector spans plotted against orbital phase and radial velocity.

pixel CCD manufactured by $\mathrm{e} 2 \mathrm{v}$, and no filter. The field of view of the instrument is $11.3^{\prime} \times 11.3^{\prime}$ and it has a plate scale of $0.66^{\prime \prime}$ pixel $^{-1}$. Autoguiding corrections are calculated directly from the science images using the DONUTS algorithm described in McCormac et al. (2013), which allows autoguiding on defocussed target stars. NITES is described in more detail in McCormac et al. (2014).

A clear transit was observed in each of the 3 follow-up nights, however data acquisition began after ingress on 2014 July 07. The middle panel of Figure 2.3 shows the three lightcurves from NITES, in bins approximately 3 minutes wide. The residuals are shown in the bottom panel. Although the beginning of some of the observations were taken at higher airmass (and consequently show larger scatter), with the simultaneous analy- 
Table 1: Radial velocity measurements of WASP135 from SOPHIE, with bisector spans from spectral line profiles.

\begin{tabular}{cccr}
\hline $\begin{array}{c}\text { BJD } \\
-240000\end{array}$ & $\begin{array}{c}\mathrm{RV} \\
\left(\mathrm{kms}^{-1}\right)\end{array}$ & $\begin{array}{c}\sigma_{R V} \\
\left(\mathrm{kms}^{-1}\right)\end{array}$ & \multicolumn{1}{c}{$\begin{array}{c}\mathrm{BS} \\
\left(\mathrm{kms}^{-1}\right)\end{array}$} \\
\hline 56808.4537 & -32.017 & 0.013 & 0.002 \\
56809.3954 & -32.678 & 0.010 & 0.022 \\
56810.4489 & -32.488 & 0.011 & -0.016 \\
56811.4723 & -32.010 & 0.009 & 0.052 \\
56812.4370 & -32.359 & 0.019 & -0.064 \\
$56813.4209^{a}$ & -32.535 & 0.015 & -0.163 \\
56814.3886 & -32.102 & 0.010 & -0.008 \\
56828.4083 & -32.137 & 0.009 & -0.000 \\
56829.4019 & -32.115 & 0.010 & -0.022 \\
56830.4209 & -32.651 & 0.009 & 0.042 \\
56856.3802 & -32.040 & 0.010 & -0.009 \\
56858.3932 & -32.653 & 0.030 & 0.177 \\
56868.4157 & -32.439 & 0.014 & -0.036 \\
56870.3629 & -32.052 & 0.009 & 0.006 \\
56897.4126 & -32.532 & 0.022 & 0.004 \\
56899.3859 & -32.246 & 0.008 & -0.015 \\
56900.3450 & -32.663 & 0.008 & 0.035 \\
56922.3090 & -32.128 & 0.008 & 0.003 \\
$56923.4023^{b}$ & -32.143 & 0.063 & -0.338 \\
56932.2772 & -32.358 & 0.010 & 0.014 \\
56933.2939 & -31.987 & 0.010 & -0.053 \\
\hline
\end{tabular}

Notes: $a$ : this measurement was a significant outlier, most likely due to instrumental effects $b$ : poor weather conditions meant that this measurement was particularly inaccurate. Neither were used in the MCMC analysis.

sis of all three light curves we are able to constrain time and shape of the ingress.

The smaller plate scale of the NITES instrument showed the presence of a brighter star on the edge of the large aperture used in the WASP photometry, and 6 fainter sources inside the aperture. This dilution meant that the transit depth in the WASP data was smaller than that found from NITES, by a factor of 2.7. Because of this, the WASP data was used to constrain the ephemeris timing only and not the transit depth. This is discussed further in Section 4

Standard data reduction was performed using PyRAF and the routines in IRAF, and DAOPHOT (Stetson 1987) was used for aperture photometry using 6 comparison stars. Since the stars were defocussed initial apertures were placed manually, then shifted for each science image using the DONUTS routine (McCormac et al. 2013).

\section{Stellar Parameters}

\subsection{Spectral Analysis}

The individual SOPHIE spectra of WASP-135 were co-added to produce a single spectrum with a $\mathrm{S} / \mathrm{N}$ of around 50 at a wavelength of $5500 \AA$. The standard pipeline reduction products were used in the analysis. The analysis was performed using the methods given in Doyle et al. (2013). The effective temperature $\left(T_{\text {eff }}\right)$ was determined from the excitation balance of the Fe I lines. The Na I $\mathrm{D}$ lines and the ionisation balance of $\mathrm{Fe}$ I and Fe II were used as surface gravity $(\log g)$ diagnostics. The parameters obtained from the analysis are listed in Table 2, The iron abundance was determined from equivalent width measurements of several unblended lines. A value for microturbulence $\left(\xi_{\mathrm{t}}\right)$ was determined from Fe I using the method of Magain (1984). The quoted error estimates include that given by the uncertainties in $T_{\text {eff }}$ and $\log g$, as well as the scatter due to measurement and atomic data uncertainties. Interstellar $\mathrm{Na} \mathrm{D}$ lines are present in the spectra with an equivalent widths of $\sim 0.06 \AA$, indicating an extinction of $E(B-V)=0.017 \pm 0.003$ using the calibration of Munari \& Zwitter (1997). Emission is detected in the $\mathrm{Ca}$ II $\mathrm{H}$ and $\mathrm{K}$ lines; however, for a star of this magnitude the SOPHIE spectra are not good enough to estimate a reliable activity index.

The projected stellar rotation velocity $(v \sin i)$ of $4.67 \pm 0.89 \mathrm{~km} \mathrm{~s}^{-1}$ was determined by fitting the profiles of several unblended Fe I lines. This agrees with the $v \sin i$ value of $4 \pm 1 \mathrm{~km} \mathrm{~s}^{-1}$ obtained from the CCF using the calibration by Boisse et al. (2010). A value for macroturbulence $\left(v_{\mathrm{mac}}\right)$ of $2.80 \pm 0.73 \mathrm{~km} \mathrm{~s}^{-1}$ was determined from the calibration of Doyle et al. (2014). An instrumental FWHM of $0.15 \pm 0.01 \AA$ was determined from the telluric lines around $6300 \AA$.

\subsection{Stellar Age Estimates}

Lithium is detected in the spectra of WASP135 , with an equivalent width of $39 \mathrm{~m} \AA$, corresponding to an abundance of $\log A(\mathrm{Li})=2.01 \pm$ 0.06 . This implies a stellar age of $0.60_{-0.35}^{+1.40} \mathrm{Gyr}$ (Sestito \& Randich 2005). 
Table 2: Stellar parameters of WASP-135 from spectroscopic analysis.

\begin{tabular}{lr}
\hline Parameter & Value \\
\hline RA & $17^{h} 49^{m} 08^{s} .4$ \\
DEC & $+29^{\circ} 52^{\prime} 45.0^{\prime \prime}$ \\
$T_{\text {eff }}$ & $5680 \pm 60 \mathrm{~K}$ \\
$\log g$ & $4.50 \pm 0.10$ \\
$v \sin i$ & $4.67 \pm 0.89 \mathrm{~km} \mathrm{~s}^{-1}$ \\
$v_{\text {mac }}$ & $2.80 \pm 0.73 \mathrm{~km} \mathrm{~s}^{-1}$ \\
$\xi_{\mathrm{t}}$ & $0.70 \pm 0.10 \mathrm{~km} \mathrm{~s}^{-1}$ \\
{$[\mathrm{Fe} / \mathrm{H}]$} & $0.02 \pm 0.13$ \\
$\log A(\mathrm{Li})$ & $2.01 \pm 0.06$ \\
Mass & $1.01 \pm 0.07 \mathrm{M}_{\odot}$ \\
Radius & $0.93 \pm 0.12 \mathrm{R}_{\odot}$ \\
Sp. Type & $\mathrm{G} 5$ \\
Distance & $300 \pm 45 \mathrm{pc}$ \\
\hline
\end{tabular}

Note: Mass and Radius estimate using the Torres et al. (2010) calibration. Spectral Type estimated from $T_{\text {eff }}$ using the table in Grav (2008). Abundances are relative to the solar values obtained by Asplund et al. (2009)

The stellar rotation rate $(P=10.08 \pm 2.32 \mathrm{~d})$ implied by the $v \sin i$ from the spectral analysis gives a gyrochronological age of $0.82_{-0.23}^{+0.41}$ Gyr using the Barnes (2010) relation. This is an upper age limit as the $v \sin i$ gives a minimum rotation rate.

Age estimates were also computed using several different stellar models for isochrone fitting using $T_{\text {eff }}$ and the stellar density $\left(\rho_{*}\right)$ from the spectral analysis and the MCMC fit respectively. The method is described in Brown (2014). The stellar data were fit to isochrones at the central metallicity of $[\mathrm{Fe} / \mathrm{H}]=0.02$, and at the upper and lower limits. These results were combined to get the final estimates of the stellar age and mass. Three sets of stellar models were used: Padova isochrones as described in Marigo et al. (2008) and Girardi et al. (2010); Yonsei-Yale isochrones as described in Demarque et al. (2004); and Dartmouth (DSEP) isochrones as described in Dotter et al. (2008). The ages calculated from these models ranged between about 3-6 Gyr, and can be found in Table 3 along with the associated stellar masses found with the models. The weighted mean and standard error of the three isochronal ages was found to be $4.4 \pm 2.5 \mathrm{Gyr}$. There is a discrepancy between the two young, fairly consistent ages from gyrochronolgy and Lithium abundances, and the older age estimates from isochrone fitting.

Table 3: Stellar age estimates for WASP-135.

\begin{tabular}{lll}
\hline Method & Age (Gyr) & Stellar Mass \\
\hline Padova isochrones & $4.21_{-6.70}^{+6.08}$ & $0.99_{-0.09}^{+0.12}$ \\
Yonsei-Yale isochrones & $3.10_{-2.56}^{+4.72}$ & $1.01_{-0.08}^{+0.08}$ \\
DSEP isochrones & $5.96_{-4.01}^{+3.91}$ & $0.95_{-0.07}^{+0.08}$ \\
Average isochronal age & $4.4 \pm 2.5$ & \\
Li abundance & $\mathbf{0 . 6 0}+1.40$ \\
Gyrochronology & $0.82_{-0.23}^{+0.41}$ \\
(upper limit) & \\
\hline $\begin{array}{l}\text { Note: Padova isochrones described in Marigo et al. } \\
\text { (2008) and Girardi et al. (2010); Yonsei-Yale isochrones }\end{array}$ \\
described in Demarque et al. (2004); Dartmouth (DSEP) \\
$\begin{array}{l}\text { isochrones described in Dotter et al. (2008); } \\
\text { Gyrochronological age from Barnes (2010) relation; Li }\end{array}$ \\
abundance age estimated from Sestito \& Randich (2005).
\end{tabular}

\section{MCMC System Parameters}

All photometry data from WASP, along with the 3 nights of NITES data and 19 radial velocity measurements from SOPHIE were used in a Markov chain Monte Carlo (MCMC) fit. However, because the photometry from WASP was diluted by other stars inside and on the edge of the aperture, the transit depth in this data set was smaller than that found with NITES by a factor of 2.7. The in-transit WASP data was therefore corrected by this factor before the MCMC fitting, as has been done for other WASP planets, for example in Anderson et al. (2012). This allowed the WASP data to constrain the ephemeris timing without affecting the transit depth.

The MCMC simultaneously fits a Keplerian orbital solution to the radial velocity data, and the Mandel \& Agol (2002) model to the photometric transits. Limb-darkening was accounted for using a non-linear model with four coefficients, which were found by interpolating from the R-band table of Claret (2000) and Claret (2004), which is appropriate for the NITES photometry.

We used an updated version of the code described in Collier Cameron et al. (2007), Pollacco et al. (2008) and Brown et al. (2012), to fit for the following parameters: the epoch of mid-transit, $\mathrm{T}_{0}$; 
the orbital period, $\mathrm{P}$; the total transit duration, $\mathrm{T}_{14}$; the ingress/egress duration, $\mathrm{T}_{12}=\mathrm{T}_{34}$; the planet to star area ratio, $R_{P}^{2} / R_{*}^{2}$; the impact parameter for a circular orbit, b; $T_{\text {eff }} ;[\mathrm{Fe} / \mathrm{H}]$, and the RV semi-amplitude, K. We applied priors on $v \sin i, T_{\text {eff }}$ and $[\mathrm{Fe} / \mathrm{H}]$ using the values listed in Table 2, and a $0.025 \mathrm{kms}^{-1}$ stellar jitter term was included in the fit. The Lucy \& Sweeney (1971) F-test was used to test for eccentricity in the Keplerian orbit. When the orbit was not forced to be circular in the MCMC fit, an eccentricity of $0.038 \pm 0.011$ was found. According to the Ftest the probability that this eccentricity could be found by chance is 0.059 , therefore a circular model is adopted in the final MCMC analysis, as recommended in Anderson et al. (2012). The resulting system parameters from this analysis are presented in Table 4.

Table 4: MCMC system parameters for WASP-135

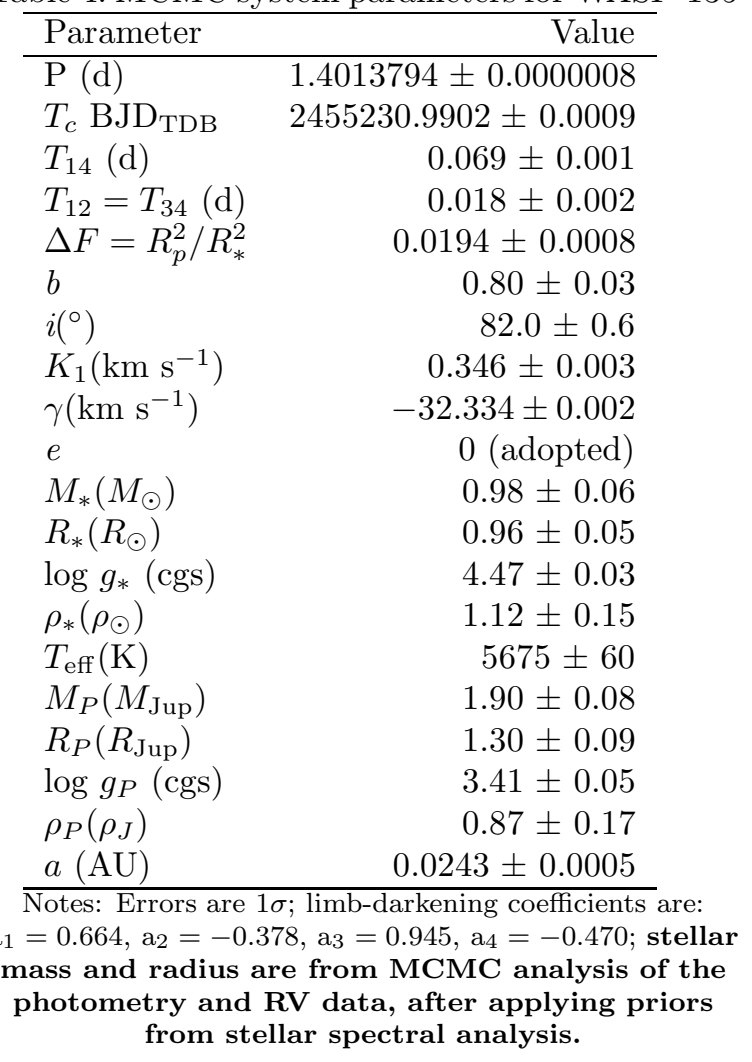

\section{Discussion}

With a large radius of $1.30 \pm 0.09 R_{\mathrm{Jup}}$, WASP$135 \mathrm{~b}$ appears to be a bloated hot Jupiter. It is a highly irradiated exoplanet, receiving an insolation of $1.98 \pm 0.24 \times 10^{9} \mathrm{erg} \mathrm{s}^{-1} \mathrm{~cm}^{-2}$, which is an order of magnitude greater than the cut off point found by Demory \& Seager (2011) of $\sim 2 \times 10^{8} \mathrm{erg}$ $\mathrm{s}^{-1} \mathrm{~cm}^{-2}$. They find that giant planets receiving more insolation than this limit have inflated radii. Additionally, Weiss et al. (2013) find strong evidence for the dependence of giant planet radius on incident flux. Their empirical relation for planet radius, mass, and incident flux predicts a radius of $1.30 \pm 0.07 R_{\mathrm{Jup}}$ for WASP-135b, which fully agrees with the value measured in this work.

The weighted mean and standard error of the three isochronal ages for WASP-135 presented here (see table 3) is $4.4 \pm 2.5 \mathrm{Gyr}$, whilst the gyrochronological age upper limit is $0.82_{-0.23}^{+0.41} \mathrm{Gyr}$. This age discrepancy could be evidence of tidal spin-up of the star, due to its massive, short period planet. On the other hand, lithium is detected in the stellar spectrum, and the age estimate of $0.60_{-0.35}^{+1.40}$ Gyr from its abundance measurement lies below the upper age limit from gyrochonological models. Since the errors on the isochronal ages are large, and the gyrochronological and lithium ages agree well, the evidence for stellar spin-up appears weak, suggesting that WASP-135 is likely a relatively young star. Of the 146 exoplanets with masses greater than $0.1 M_{\text {Jup }}$, orbital distances less than $0.1 \mathrm{AU}$, and published estimates for their stellar host age, only 7 orbit stars younger than 0.8 Gyı! 1 .

The authors would like to thank the anonymous referee for their valuable comments which greatly improved this paper. The WASP Consortium consists of representatives from the Universities of Keele, Leicester, The Open University, Queens University Belfast, St Andrews and Warwick, along with the Isaac Newton Group (La Palma) and the Instituto de Astrofisica de Canarias (Tenerife). SuperWASP-North is hosted by the Isaac Newton Group and the Instituto de Astrofisica de Canarias; we gratefully acknowledge their ongoing support and assistance. The SuperWASP cameras are operated with funds available from Consortium Universities and the STFC. The SOPHIE observations were made possible thanks to OPTICON funding allocations 2014A/043 (PI

\footnotetext{
${ }^{1}$ exoplanetarchive.ipac.caltech.edu
} 
Faedi) and 2014B/029 (PI Gómez Maqueo Chew). YGMC has been partially funded by Programa UNAM-DGAPA-PAPIIT IA-103215. This research has made use of NASA's Astrophysics Data System Bibliographic Services.

Facilities: SuperWASP, OHP:1.93m (SOPHIE), NITES.

\section{REFERENCES}

Anderson, D. R., Collier Cameron, A., Gillon, M., et al. 2012, MNRAS, 422, 1988

Asplund, M., Grevesse, N., Sauval, A. J., \& Scott, P. 2009, ARA\&A, 47, 481

Baraffe, I., Chabrier, G., \& Barman, T. 2010, Reports on Progress in Physics, 73, 016901

Baranne, A., Queloz, D., Mayor, M., et al. 1996, A\&AS, 119, 373

Barnes, S. A. 2010, ApJ, 722, 222

Batygin, K. \& Stevenson, D. J. 2010, ApJ, 714, L238

Boisse, I., Eggenberger, A., Santos, N. C., et al. 2010, A\&A, 523, A88

Bouchy, F., Hébrard, G., Udry, S., et al. 2009, A\&A, 505, 853

Brown, D. J. A. 2014, MNRAS, 442, 1844

Brown, D. J. A., Collier Cameron, A., Díaz, R. F., et al. 2012, ApJ, 760, 139

Claret, A. 2000, A\&A, 363, 1081

Claret, A. 2004, A\&A, 428, 1001

Collier Cameron, A., Pollacco, D., Street, R. A., et al. 2006, MNRAS, 373, 799

Collier Cameron, A., Wilson, D. M., West, R. G., et al. 2007, MNRAS, 380, 1230

Demarque, P., Woo, J.-H., Kim, Y.-C., \& Yi, S. K. 2004, ApJS, 155, 667

Demory, B.-O. \& Seager, S. 2011, ApJS, 197, 12

Dotter, A., Chaboyer, B., Jevremović, D., et al. 2008, ApJS, 178, 89
Doyle, A. P., Davies, G. R., Smalley, B., Chaplin, W. J., \& Elsworth, Y. 2014, MNRAS, 444, 3592

Doyle, A. P., Smalley, B., Maxted, P. F. L., et al. 2013, MNRAS, 428, 3164

Eastman, J., Siverd, R., \& Gaudi, B. S. 2010, PASP, 122, 935

Enoch, B., Cameron, A. C., Anderson, D. R., et al. 2011, MNRAS, 410, 1631

Fortney, J. J. \& Nettelmann, N. 2010, Space Sci. Rev., 152, 423

Girardi, L., Williams, B. F., Gilbert, K. M., et al. 2010, ApJ, 724, 1030

Gray, D. F. 2008, The observation and analysis of stellar photospheres, 3rd edn. (Cambridge University Press), p. 507

Guillot, T. \& Showman, A. P. 2002, A\&A, 385, 156

Lanza, A. F. 2010, A\&A, 512, A77

Lucy, L. B. \& Sweeney, M. A. 1971, AJ, 76, 544

Magain, P. 1984, A\&A, 134, 189

Mandel, K. \& Agol, E. 2002, ApJ, 580, L171

Marigo, P., Girardi, L., Bressan, A., et al. 2008, A\&A, 482, 883

Maxted, P. F. L., Anderson, D. R., Collier Cameron, A., et al. 2011, PASP, 123, 547

Maxted, P. F. L., Serenelli, A. M., \& Southworth, J. 2015, ArXiv e-prints

Mayor, M. \& Queloz, D. 1995, Nature, 378, 355

McCormac, J., Pollacco, D., Skillen, I., et al. 2013, PASP, 125,548

McCormac, J., Skillen, I., Pollacco, D., et al. 2014, MNRAS, 438, 3383

Munari, U. \& Zwitter, T. 1997, A\&A, 318, 269

Pepe, F., Mayor, M., Galland, F., et al. 2002, A\&A, 388, 632

Pollacco, D., Skillen, I., Collier Cameron, A., et al. 2008, MNRAS, 385, 1576 
Pollacco, D. L., Skillen, I., Collier Cameron, A., et al. 2006, PASP, 118, 1407

Pont, F. 2009, MNRAS, 396, 1789

Queloz, D., Henry, G. W., Sivan, J. P., et al. 2001, A\&A, 379, 279

Sestito, P. \& Randich, S. 2005, A\&A, 442, 615

Showman, A. P. \& Guillot, T. 2002, A\&A, 385, 166

Smith, A. M. S., Hebb, L., Collier Cameron, A., et al. 2009, MNRAS, 398, 1827

Spiegel, D. S. \& Burrows, A. 2013, ApJ, 772, 76

Stetson, P. B. 1987, PASP, 99, 191

Toner, C. G. \& Gray, D. F. 1988, ApJ, 334, 1008

Torres, G., Andersen, J., \& Giménez, A. 2010, A\&A Rev., 18, 67

Weiss, L. M., Marcy, G. W., Rowe, J. F., et al. 2013, ApJ, 768, 14

Zacharias, N., Finch, C. T., Girard, T. M., et al. 2013, AJ, 145, 44

This 2-column preprint was prepared with the AAS LATEX macros v5.2. 